\title{
Acumulación De Restos Lignino-Celulósicos (Necromasa) En Un Bosque Templado De Antiguo Crecimiento Dominado Por Nothofagus En El Centro- Sur De Chile
}

Carlos Belezaca Pinargote

Darwin Salvatierra Piloso

Diana Delgado Campusano

Universidad Técnica Estatal de Quevedo,

Facultad de Ciencias Ambientales. Quevedo, Ecuador

Roberto Godoy Bórquez

Eduardo Valenzuela Flores

Universidad Austral de Chile, Facultad de Ciencias. Valdivia, Chile.

Edwin Jiménez Romero

Rolando López Tobar

Renato Baque Mite

Roberto Herrera Anangono

Universidad Técnica Estatal de Quevedo,

Facultad de Ciencias Ambientales. Quevedo, Ecuador

Byron Oviedo Bayas

Universidad Técnica Estatal de Quevedo,

Facultad de Ciencias de la Ingeniería. Quevedo, Ecuador

María Cadme Arevalo

Universidad Técnica Estatal de Quevedo,

Facultad de Ciencias Ambientales. Quevedo, Ecuador

Tito Bohórquez Barros

Carmen Vásconez Montufar

Universidad Técnica de Babahoyo,

Carrera de Ingeniería Agropecuaria. Babahoyo, Ecuador

URL:http://dx.doi.org/10.19044/esj.2016.v12n9p69

Abstract
Coarse woody debris (CWD) are the necromass in wooded
environments and comply with various ecosystems functions, such as
seedling nursery, habitat other organisms, store carbon (C) and nutrients,
etc.. Volume, necromass, and decay states of CWD dead in an old-growth 
temperate (OGTF) forest in Puyehue National Park, South-Central Chile were evaluated. In 10 plots of $900 \mathrm{~m}^{2} \mathrm{CWD}$ was quantified $(\geq 10 \mathrm{~cm}$ diameter), whose necromass classified using a scale of five categories/stages of decay, necromass $(1=$ lowest and $5=$ highest degradation). The average forest density was 299 trees $\mathrm{ha}^{-1}, 112 \mathrm{~m}^{2} \mathrm{ha}^{-1}$ of basal area, and $2.395 \mathrm{~m}^{3}$ of stem volume. The upper arboreal stratum was dominated by $N$. betuloides . The greatest amount of CWD belonged to $N$. betuloides $(95,2 \%)$, where logs $(52,7 \%)$ and branches $(35 \%)$, plant structures were present in greater numbers. The bulk of necromass found in advanced states of decomposition (level 4 and 5) with 56,5\% and 34,1\%, respectively. It was determined that the CWD volume was $632 \mathrm{~m}^{3} \mathrm{ha}^{-1}$, representing a necromass of $321.5 \mathrm{Mg} \mathrm{ha}^{-}$ 1. These results demonstrate that the old-growth temperate forests of southern Chile are significant reserves of coarse woody debris, which contributes to the biogeochemistry of these complex and remote ecosystems.

Keywords: Biogeochemical, decaying wood, dead wood, logs, Puyehue National Park

\section{Resumen}

Los restos lignino-celulósicos de gran tamaño (RLC), constituyen la necromasa en ambientes boscosos, y cumplen variadas funciones ecosistémicas, como vivero para plántulas, hábitat de otros organismos, almacén de carbono (C) y nutrientes, etc. Se evaluó el volumen, biomasa y grado de descomposición de RLC depositados un bosque templado de antiguo crecimiento del Parque Nacional Puyehue, Centro-Sur de Chile. Se delimitaron 10 parcelas de $900 \mathrm{~m}^{2}$, donde se cuantificó, midió (DAP y altura) y estratifico la vegetación viva, e identificó, clasificó, cuantificó y midió (diámetro y longitud) $\operatorname{los} \mathrm{RLC} \geq 10 \mathrm{~cm}$ de diámetro. Una escala de cinco categorías/estados de descomposición se usó para clasificar los RLC, siendo $1=$ menor y 5 = mayor descomposición. La densidad promedio del bosque fue de 299 árboles ha ${ }^{-1}$, con $112 \mathrm{~m}^{2} \mathrm{ha}^{-1}$ de área basal, y $2.395 \mathrm{~m}^{3} \mathrm{de}$ volumen fustal. El estrato arbóreo superior lo dominó Nothofagus betuloides. La mayor cantidad de RLC pertenecieron a $N$. betuloides $(95,2 \%)$, siendo troncos $(52,7 \%)$ y ramas $(35 \%)$ las estructuras vegetales existentes en mayor número. La mayor parte de necromasa se encontró en avanzados estados de descomposición (escala 4 y 5) con 56,5\% y 34,1\%, respectivamente. Se determinó que el volumen de RLC fue de $632 \mathrm{~m}^{3} \mathrm{ha}^{-1}$, lo que representó una necromasa de $321,5 \mathrm{Mg} \mathrm{ha}^{-1}$. Estos resultados demuestran que los bosques templados de antiguo crecimiento del Centro-Sur de Chile son importantes reservas de necromasa, y de biomasa viva. 
Palabras clave: Biogeoquímica, descomposición de madera, madera muerta, Parque Nacional Puyehue, troncos

\section{Introducción}

Los bosques templados de antiguo crecimiento, localizados en las estribaciones andinas del cono sur de Suramérica, compartidos por Chile y Argentina, representan ecosistemas particulares por la composición e historia de su biota (Donoso y Lusk, 2007; Veblen et al., 2011), donde su estabilidad y permanencia depende casi exclusivamente de la circulación interna de nutrientes, a través de la descomposición y mineralización de la producción primaria neta (PPN) que después pasa a formar parte de la materia orgánica del suelo (MOS), (Huygens et al., 2008; Godoy et al., 2009).

La PPN de estos complejos ecosistemas constituye la clave para su eficiente ciclaje, donde los constantes aportes de litera fina lábil al suelo (hojarasca, flores, frutos, etc.), suministran nutrientes a corto plazo (Decker y Boerner, 2006; Ibarra et al., 2011), mientras que la litera leñosa gruesa, formada por restos lignino-celulósicos de mayor tamaño (RLC), (troncos, tocones, ramas, etc.), constituyen una fuente de nutrientes como capital a largo plazo, mediada por una liberación más lenta y gradual (Heinemann y Kitzberger, 2006). La acumulación de RLC sobre y bajo el piso forestal, ocurre como producto de disturbios naturales (bosques prístinos) y antropogénicos (bosques intervenidos), (Carmona et al., 2002; Schlegel y Donoso, 2008). En bosques prístinos su presencia es evidente, constituyendo un componente funcional y estructural como hábitat para productores $\mathrm{y}$ consumidores (Jia-bing et al., 2005) que tributan a la biogeoquímica del sistema (Heinemann y Kitzberger, 2006).

En bosques templados maduros y de lento crecimiento del sur de Chile, se ha estimado que la mayor parte de restos lignino-celulósicos (86 a 94\% del total) que forman la necromasa gruesa (Carmona et al., 2002; Schlegel y Donoso, 2008) observada en el presente, pertenecen a árboles muertos hace varias décadas (Césari et al., 2010; Lombardi et al., 2011), y su relevancia es evidente en las fases de desarrollo del bosque (Césari et al., 2010). Entre las contribuciones de esta necromasa, se destacan la función de sustrato para plántulas de regeneración del bosque, hábitat para meso y microbiota, entre otros (Brown et al., 2013). Su descomposición implica procesos físicos y bioquímicos importantes dentro del reciclaje en ecosistemas terrestres, no solo por el aporte de nutrientes al suelo y formación de materia orgánica (González-Polo et al., 2013), sino también por su contribución al balance global de C (Gough et al., 2007).

Si bien, existe escasa información sobre RLC en bosques templados de antiguo crecimiento del sur de Chile (Carmona et al., 2002; Schlegel y Donoso, 2008), observaciones de campo dan cuenta de la presencia de estos 
elementos en diferentes estados de descomposición, que probablemente perduran por años y decenios, fraccionándose y mineralizándose lentamente, contribuyendo a los procesos biogeoquímicos del ecosistema (Lombardi et al., 2011). En este sentido, el ciclaje de nutrientes desde los RLC, estaría en función de las etapas de descomposición, definidas de acuerdo a sus características físico-químicas, generalmente proporcionadas por la antigüedad de la madera en el suelo (Spies et al., 1988; Marra y Edmonds, 1994; Yan et al., 2006).

De acuerdo a los antecedentes antes mencionados, en el presente trabajo se planteó: 1) determinar cuali-cuantitativamente la necromasa gruesa lignino-celulósica ( $\geq 10 \mathrm{~cm}$ de diámetro) acumulada en un bosque templado de antiguo crecimiento del Centro-Sur de Chile.

\section{Materiales y métodos}

Área de estudio y establecimiento de parcelas. Estuvo ubicada en la Cordillera de Los Andes, en una formación vegetal de bosque templado siempreverde de antiguo crecimiento, dentro del Parque Nacional Puyehue, Centro-Sur de Chile $\left(40^{\circ} 47^{\prime} \mathrm{S}-72^{\circ} 12^{\prime} \mathrm{O}\right)$. El clima es lluvioso con una precipitación promedio, superior a $7000 \mathrm{~mm}$ anuales y una temperatura media anual del aire de $4,5{ }^{\circ} \mathrm{C}$ (Oyarzún et al., 2004). Se establecieron 10 parcelas de 30 × $30 \mathrm{~m}\left(900 \mathrm{~m}^{2}\right)$ en el bosque, donde habitan las especies forestales Nothofagus betuloides (Mirb.) Oerst (coigue), Saxegothaea conspicua Lindl. (mañio hembra) y Laureliopsis philippiana (Looser) R. Schodde (tepa), con individuos que poseen edades superiores a los 300 años.

Estratificación del dosel. En cada parcela se registró el porcentaje de cobertura de 5 estratos horizontales del bosque, siguiendo una categorización arbitraria y registró las especies vegetales presentes en cada estrato (Cuadro 1).

Cuadro 1. Categorías empleadas para la estratificación en parcelas de un bosque templado siempreverde de antiguo crecimiento, Parque Nacional Puyehue, Centro-Sur de Chile.

\begin{tabular}{cc}
\hline CATEGORÍA DE ESTRATOS & DISCRIMINACIÓN POR ALTURA \\
\hline Estrato Arbóreo Superior & Hasta 45 metros \\
\hline Estrato Arbóreo Medio & Hasta 25 metros \\
\hline Estrato Arbóreo Bajo & Hasta 10 metros \\
\hline Estrato Arbustivo & Hasta 4 metros \\
\hline Estrato herbáceo & $<1$ metro
\end{tabular}

\section{Volumen y área basal de árboles en pie}

Durante los meses de octubre y noviembre del año 2013, con la asistencia de una forcípula e hipsómetro Sunnto, se estimó el DAP (diámetro a la altura del pecho, 1,30 m) y la altura en los árboles existentes en cada 
parcela, respectivamente. Con los datos de campo, se procedió a calcular el volumen para cada especie forestal, empleando la fórmula para árboles en pie [1], y él área basal mediante la fórmula [2]. Posteriormente, los resultados se extrapolaron a $\mathrm{m}^{3}$ (volumen) y/o $\mathrm{m}^{2}$ (área basal) por hectárea.

$$
\begin{array}{ccc}
V=\frac{\pi *(D A P)^{2}}{4} * L * F f & \\
\text { Donde: } & = & \text { Volumen }\left(\mathrm{m}^{3}\right) . \\
\pi & = & 3,1416 . \\
\text { DAP } & = & \text { Diámetro a la altura del pecho }(1,30 \mathrm{~m}) . \\
\mathrm{L} & = & \text { Altura }(\mathrm{m}) . \\
\mathrm{Ff} & = & \text { Factor de forma. } \\
& & \\
A B=\frac{\pi *(D A P)^{2}}{4} & & \text { Área basal }\left(\mathrm{m}^{2}\right) . \\
\text { Donde: } & & 3,1416 . \\
\pi & = & \text { Diámetro a la altura del pecho }(1,30 \mathrm{~m}) .
\end{array}
$$

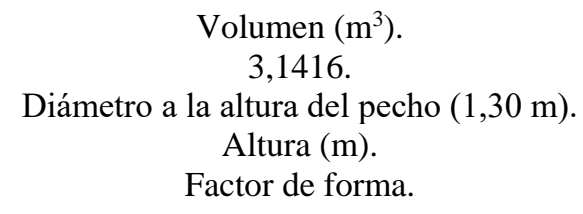

\section{Estimación del volumen y necromasa lignino-celulósica}

Se midió el diámetro (mayor, medio, y menor), y longitud de los RLC ( $\geq 10 \mathrm{~cm}$ de diámetro), e identificó las especies forestales a quien pertenecían. Posteriormente, los datos se procesaron, analizaron y mediante la fórmula de Smaliam [3] sugerida para cubicar trozas apeadas, se estimó el volumen de restos leñosos. Los RLC se clasificados en 5 categorías, de acuerdo a sus características físicas y grado de descomposición, siguiendo los criterios propuestos por Spies et al. (1988), Marra y Edmonds (1994), Yan et al. (2006), (Cuadro 2). La biomasa seca, fue determinada multiplicando el volumen de cada sección de madera, por la densidad específica $\left(\mathrm{g}^{-1} \mathrm{~cm}^{-3}\right)$, como lo realizó Gayoso (2001) en especies forestales de bosques siempreverdes de Chile, similares a las del presente estudio. Se utilizó los valores de densidad 0,$51 ; 0,44 ; 0,36 ; 0,31 ;$ y $0,25 \mathrm{~g}^{-1} \mathrm{~cm}^{3}$ asignados a cada una de las cinco categorías de descomposición, respectivamente.

$$
\begin{aligned}
& \mathrm{V}=\left(\frac{\pi}{4}\right) *\left(\frac{D_{\text {mayor }}+D_{\text {medio }}+D_{\text {menor }}}{3}\right)^{2} * L \\
& \text { Donde: } \\
& \mathrm{V}= \\
& \pi \quad= \\
& \mathrm{D} \quad= \\
& \mathrm{L} \quad=
\end{aligned}
$$

Volumen $\left(\mathrm{m}^{3}\right)$. 3,1416 .

Diámetro $(\mathrm{m})$.

Longitud (m). 
Cuadro 2. Clasificación de restos lignino-celulósicos gruesos de acuerdo a los criterios sugeridos por Spies et al. (1988), Marra y Edmonds (1994), y Yan et al. (2006).

\begin{tabular}{|c|c|}
\hline CATEGORÍAS & CARACTERÍSTICAS \\
\hline 1 & Material recientemente caído con tejidos y corteza intactos. \\
\hline 2 & $\begin{array}{l}\text { Albura empezando a descomponerse, pero completamente presente. } \\
\text { Corteza empezando a romperse. }\end{array}$ \\
\hline 3 & Albura y corteza mayormente presente, duramen intacto. \\
\hline 4 & $\begin{array}{c}\text { Corteza y albura casi desaparecida, duramen empezando a } \\
\text { descomponerse. }\end{array}$ \\
\hline 5 & $\begin{array}{l}\text { Albura y corteza desaparecido, duramen con avanzado estado de } \\
\text { descomposición. }\end{array}$ \\
\hline
\end{tabular}

Análisis estadísticos. Los datos obtenidos por cada variable estudiada, se sometieron a un análisis de comprobación de los supuestos de normalidad y homocedasticidad de varianzas. Finalmente, mediante estadística descriptiva se obtuvieron los promedios y errores estándar. Todos los análisis estadísticos se efectuaron en el programa STATISTICA 7, versión para Windows.

\section{Resultados}

Dasonomía y estratificación del bosque. Se determinó que $N$. betuloides, $S$. conspicua y $L$. philippiana fueron las especies forestales más representativas en las 10 parcelas estudiadas, llegando a dominar los dos primeros estratos superiores del bosque, con una densidad promedio de 299 árboles ha ${ }^{-1}$ (rango 189 a 433 árboles ha ${ }^{-1}$ ), que representaron $112 \mathrm{~m}^{2} \mathrm{ha}^{-1}$ de área basal (rango 84,5 a 146,3 $\mathrm{m}^{2} \mathrm{ha}^{-1}$ ), y un volumen promedio de 2394,9 $\mathrm{m}^{3} \mathrm{ha}^{-1}$ (rango $1.566,2$ y $3.252,1 \mathrm{~m}^{3} \mathrm{ha}^{-1}$ ), (Cuadro 3 ).

Cuadro 3. Variables dasonómicas estimadas en 10 parcelas experimentales de un bosque templado siempreverde de antiguo crecimiento, Parque Nacional Puyehue, Centro-Sur de Chile.

\begin{tabular}{cccc}
\hline Nro. de parcelas & Densidad (árboles ha $\left.{ }^{-1}\right)$ & Área basal $\left(\mathrm{m}^{2}\right.$ ha & Volumen $\left(\mathrm{m}^{3} \mathrm{ha}^{-1}\right)$ \\
\hline 1 & 200 & 110,3 & $2.247,8$ \\
\hline 2 & 344 & 146,3 & $3.250,9$ \\
\hline 3 & 189 & 138,0 & $3.252,1$ \\
\hline 4 & 244 & 84,5 & $1.566,2$ \\
\hline 5 & 278 & 131,9 & $3.087,9$ \\
\hline 6 & 344 & 105,1 & $2.515,7$ \\
\hline 7 & 300 & 105,2 & $2.076,9$ \\
\hline 8 & 189 & 101,5 & $2.140,5$ \\
\hline 9 & 433 & 110,2 & $2.169,1$ \\
\hline 10 & 467 & 86,7 & $1.642,2$ \\
\hline Promedio & $299( \pm 31,2)^{*}$ & $112( \pm 6,6)^{*}$ & $2.394,9( \pm 195,9)^{*}$ \\
\hline \multicolumn{4}{c}{}
\end{tabular}

\footnotetext{
* Error estándar
} 


\section{Caracterización cuali-cuantitativa de restos lignino-celulósicos}

La necromasa lignino-celulósica encontrada en el bosque perteneció principalmente a $N$. betuloides (95\%), y en menor cantidad a $S$. conspicua (3,7\%), L. phillipiana (0,8\%) y Dasyphyllum diacanthoides (Less.) Cabrera $(0,3 \%)$. En la figura 1 se muestran los aportes $\left(\mathrm{m}^{3} \mathrm{ha}^{-1}\right)$ de RLC.

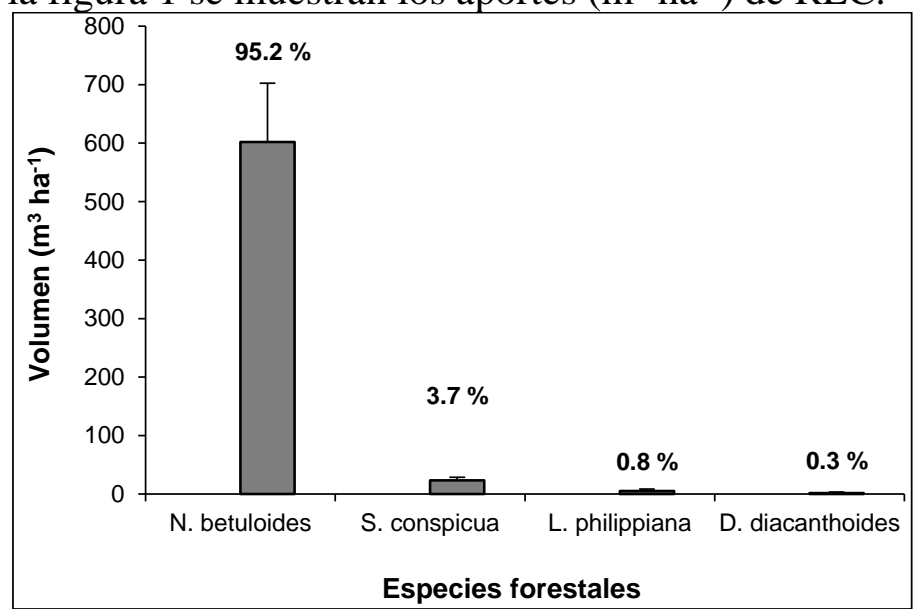

Figura 1. Volumen $\left(\mathrm{m}^{3} \mathrm{ha}^{-1}\right)$ de restos lignino-celulósicos depositados en un bosque templado siempreverde de antiguo crecimiento, Parque Nacional Puyehue, Centro-Sur de Chile. Valores corresponden a promedios de 10 parcelas con su respectivo error estándar.

Los RLC se contabilizaron y clasificaron de acuerdo a la sección del árbol que pertenecían, considerándose ramas, troncos, tocones depositados en el suelo, y árboles muertos en pie. El número promedio de restos leñosos fue de 378 unidades por $\mathrm{ha}^{-1}$. La mayor cantidad de restos pertenecieron a ramas y troncos, con promedios generales de 142 y 192 unidades, representando el 35 y $52,7 \%$ de la necromasa por $\mathrm{ha}^{-1}$, respectivamente. El número promedio de tocones y árboles muertos en pie, fue menor, alcanzando valores de 20 unidades $(5,59 \%)$, y 23 unidades $(6,67 \%)$ por ha ${ }^{-1}$, respectivamente (Figura 2).

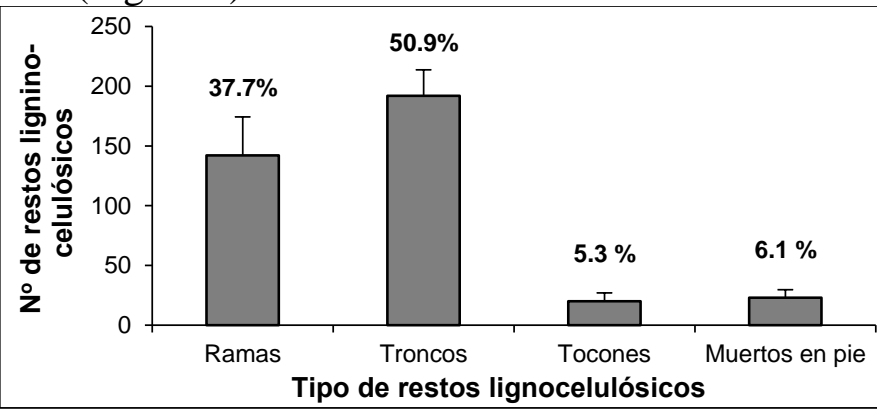

Figura 2. Número, tipo y porcentaje de restos lignino-celulósicos por $\mathrm{ha}^{-1}$, encontrados en un bosque templado siempreverde de antiguo crecimiento, Parque Nacional Puyehue, Centro-

Sur de Chile. Valores corresponden a promedios de 10 parcelas con su respectivo error estándar. 
Se determinó que la mayor parte de RLC se encontraban en los dos últimos estados de descomposición 4 y 5 (según escala), con 216 y 126 unidades por $\mathrm{ha}^{-1}$ como promedio, representando el 57,7 y 33\%, respectivamente. La presencia de restos leñosos en las categorías 1, 2 y 3, fue de baja representatividad, encontrándose como promedios 9, 7, y 20 unidades por $\mathrm{ha}^{-1}$, lo cual constituye apenas el 2,4; 1,6; y 5,3\%, respectivamente (Figura 3).

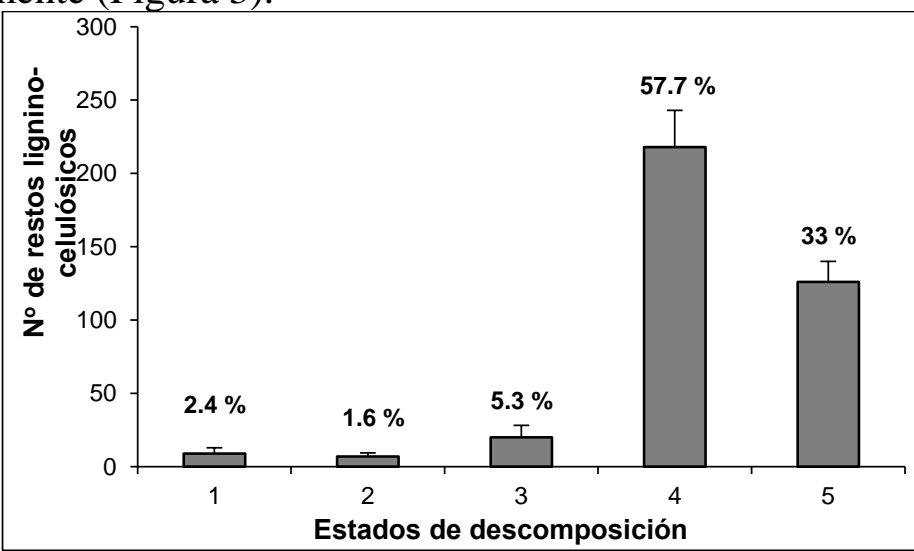

Figura 3. Número y porcentaje de restos lignino-celulósicos por categoría de descomposición, contabilizados en un bosque templado siempreverde de antiguo crecimiento, Parque Nacional Puyehue, Centro-Sur de Chile. Valores corresponden a promedios de 10 parcelas con su respectivo error estándar.

En total se cuantificaron $632 \mathrm{~m}^{3} \mathrm{ha}^{-1}$ de volumen, que representaron 231,5 $\mathrm{Mg}^{-1} \mathrm{ha}^{-1}$ de necromasa. La mayor parte de la necromasa se encontró en estados avanzados de descomposición (escala 4 y 5), con $375,7 \mathrm{~m}^{3} \mathrm{ha}^{-1}$ (146,5 $\left.\mathrm{Mg} \mathrm{ha}^{-1}\right)$, y $181,7 \mathrm{~m}^{3} \mathrm{ha}^{-1}\left(49,1 \mathrm{Mg} \mathrm{ha}^{-1}\right)$, respectivamente (Figura 4).

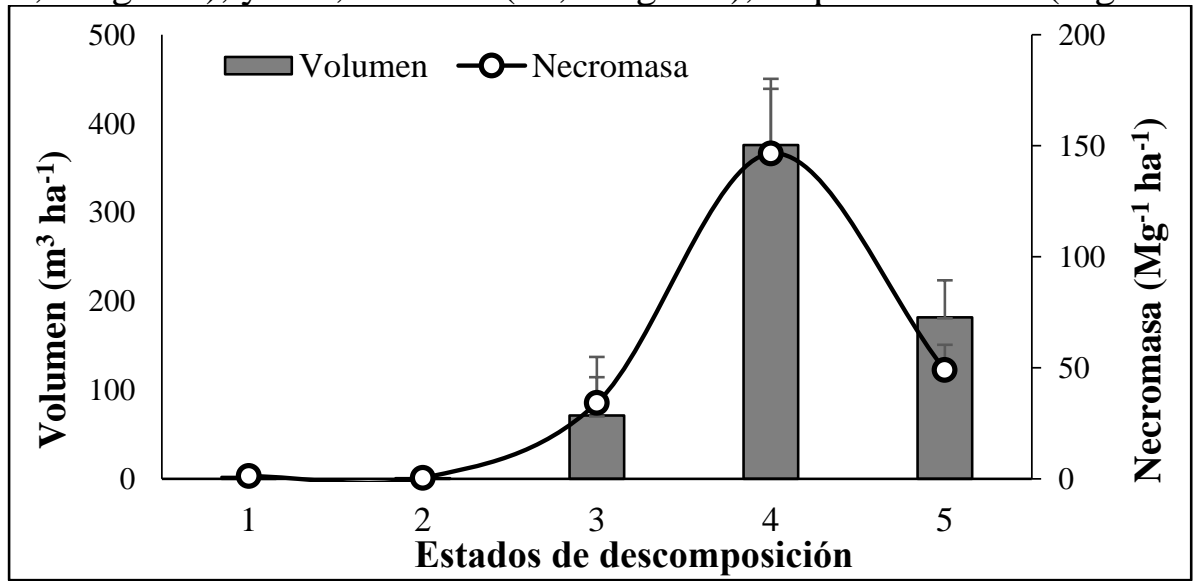

Figura 4. Volumen $\left(\mathrm{m}^{3} \mathrm{ha}^{-1}\right)$ y necromasa $\left(\mathrm{Mg} \mathrm{ha}^{-1}\right)$ en cinco estados de descomposición, bajo un bosque templado siempreverde de antiguo crecimiento, Parque Nacional Puyehue, Centro-Sur de Chile. Valores corresponden a promedios de 10 parcelas con su respectivo error estándar. 


\section{Discusión}

Densidad, área basal, volumen y estratificación del bosque. Los valores promedio de las variables densidad (299 árboles ha ${ }^{-1}$ ), área basal $\left(112 \mathrm{~m}^{2} \mathrm{ha}^{-1}\right)$ y volumen $\left(2395 \mathrm{~m}^{3} \mathrm{ha}^{-1}\right)$, encontrados en un bosque templado siempreverde de antiguo crecimiento del Parque Nacional Puyehue, estuvieron dentro del rango descrito para este tipo de bosques maduros del Centro-Sur de Chile (Donoso, 2013). Sin embargo, estos valores están por debajo de los reportados por Almonacid (2006) y Schlegel y Donoso (2008), quienes en un bosque de similares características en San Pablo de Tregua (39 35' $\left.\mathrm{S}-72^{\circ} 04^{\prime} \mathrm{O}\right)$, encontraron rangos de densidad de 418 - 501 árboles $\mathrm{h}^{-1}$ y $114,16-125,6 \mathrm{~m}^{2} \mathrm{ha}^{-1}$ de área basal, respectivamente. Por otra parte, Carmona et al. (2002) en bosques templados de antiguo crecimiento en Chiloé $\left(42^{\circ} 30^{\prime} \mathrm{S}\right)$, reportó rangos de densidades $\left(2708-3250\right.$ árboles $\left.^{-1}\right)$, $\mathrm{y}$ área basal $\left(68,9-109,4 \mathrm{~m}^{2} \mathrm{ha}^{-1}\right)$, cuyos valores fueron superiores $\mathrm{e}$ inferiores, respectivamente, a los encontrados en la presente investigación. Estas variaciones estarían ligadas a los diferentes tipos de asociaciones vegetales, estados sucesionales y heterogeneidad propia de los bosques templados del sur de Chile, desarrollados bajo condiciones extremas, como bajas temperaturas, precipitaciones superiores a los $7000 \mathrm{~mm}$ anuales, limitaciones nutricionales, e intermitente actividad volcánica, que ejerce cambios en la estructura vegetacional (Godoy et al., 2009).

\section{Restos lignocelulósicos}

El volumen (632 $\left.\mathrm{m}^{3} \mathrm{ha}^{-1}\right)$ y necromasa $\left(231,5 \mathrm{Mg} \mathrm{ha}^{-1}\right)$ de RLC en descomposición, muestran que el bosque templado de antiguo crecimiento estudiado, posee cantidades significativas de madera muerta, donde los mayores aportes fueron de $N$. betuloides con el $95,2 \%$, respecto a $S$. conspicua, L. philippiana y D. diacanthoides, que tuvieron una participación marginal. El volumen de los RLC depositados en el bosque, representó el $16,4 \%$ del volumen fustal de los árboles vivos por $\mathrm{ha}^{-1}$. Los resultados obtenidos reflejan la dominancia de $N$. betuloides, especie sombraintolerante, cuyos restos maderables depositados en el suelo, liberan espacios con acceso a luz, que rápidamente son colonizados por la regeneración natural, contribuyendo a una sucesión gradual de la vegetación y un lento reciclaje de nutrientes (Watkinson et al., 2006). Bajo condiciones prístinas, los restos maderables se acumulan en el suelo, cuando el bosque sufre disturbios naturales recurrentes, como actividad volcánica, vientos, nevadas, lluvias torrenciales, derrumbes, o en su defecto ataques de plagas, especialmente en árboles que han llegado al final de su ciclo biológico (Brown et al., 2013).

La mayor parte de los RLC depositados en el suelo del bosque (632 $\mathrm{m}^{3} \mathrm{ha}^{-1} \approx 231,5 \mathrm{Mg} \mathrm{ha}^{-1}$ ) se encontraron en los estados más avanzados de 
descomposición (4 y 5), lo cual indicaría que el bosque es de crecimiento antiguo, y la madera que actualmente se encuentra en degradación, sería el resultado de disturbios naturales ocurridos hace varias décadas, concordante con lo señalado por Césari et al. (2010) y Lombardi et al. (2011). Bajo estas condiciones, la madera en degradación avanzada, constituye un lugar de intensa actividad microbiológica, principalmente fúngica (Dill y Kraepelin, 1986), que actúan en la transformación y aprovechamiento relativamente rápido de elementos lábiles, (almidones, pectina, proteínas) y luego en la depolimerización gradual de biomoléculas más complejas, como celulosa y lignina (Blanchette, 1991).

\section{Conclusion}

- $\quad$ El estrato superior del área de bosque templado siempreverde de antiguo crecimiento estudiado, estuvo dominada $N$. betuloides, y en los estratos intermedios por la combinación de $N$. betuloides, $S$. conspicua y $L$. phillipiana.

- La mayor cantidad de restos lignocelulósicos acumulados en el bosque templado de antiguo crecimiento, pertenecieron a $N$. betuloides $(95,2 \%)$, siendo los troncos $(52,7 \%)$ y ramas $(35 \%)$ las estructuras vegetales con mayor presencia en número, volumen y necromasa.

- El bosque templado de antiguo crecimiento dominado por Nothofagus, almacena $632 \mathrm{~m}^{3} \mathrm{ha}^{-1}$ de restos lignino-celulósicos, que representaron $231,5 \mathrm{Mg} \mathrm{ha}^{-1}$ de necromasa, cuyos restos se encuentran en los estados $4(56,5 \%)$ y $5(34,1 \%)$ de descomposición, indicando que la mayor parte de estos materiales orgánicos, están en degradación avanzada.

\section{References:}

Almonacid, L.J. 2006. Mineralización de nitrógeno en bosques siempreverdes de la cordillera de los Andes, sur de Chile. Tesis de Grado de Licenciado en Ciencias Biológicas. Facultad de Ciencias, Universidad Austral de Chile. 43 p.

Blanchette, R.A. 1991. Delignification by wood-decay fungi. Annual Review of Phytopathology 29: 381-398.

Brown, M.J., Kertis, J., y Huff, M.H. 2013. Natural Tree Regeneration and Coarse Woody Debris Dynamics After a Forest Fire in the Western Cascade Range. USDA, USA. $50 \mathrm{p}$.

Carmona, M.R., Armesto, J.J., Aravena, J.C., y Pérez, C.A. 2002. Coarse woody debris biomass in successional and primary temperate forests in Chiloé Island, Chile. Forest Ecology and Management, 164(1-3): 265-275.

Césari, S.N., Busquets, P., Colombo Piñol, F., Méndez Bedia, I., y Limarino, C.O. 2010. Nurse logs: An ecological strategy in a late Paleozoic forest from the southern Andean region. Geology, 38(4): 295-298. 
Decker KLM, REJ Boerner. 2006. Mass loss and nutrient release from decomposing evergreen and deciduous Nothofagus litters from the Chilean Andes. Austral Ecology, 31(8): 1005-1015.

Dill, I., y Kraepelin, G. 1986. Palo Podrido: Model for extensive delignification of Wood by Ganoderma applanatum. Applied and Environmental Microbiology 52(6):1305-1312.

Donoso PJ, CH Lusk. 2007. Differential effects of emergent Nothofagus dombeyi on growth and basal area of canopy species in an old-growth temperate rainforest. Journal of Vegetal Science 18: 675-684.

Donoso, P.J. 2013. Necesidades, opciones y futuro del manejo multi-etáneo en el centro-sur de Chile. In Silvicultura en Bosques Nativos. Avances en la investigación en Chile, Argentina y Nueva Zelandia. Estudios en Silvicultura de Bosques Nativos Vol. 1. Donoso P, Promis A. (eds). pp 5-85.

Gayoso, J. 2001. Medición de la capacidad de captura de carbono en bosques nativos y plantaciones de Chile. Revista Forestal Iberoamericana, 1(1): 113.

Godoy, R., Paulino, L., Valenzuela, E., Oyarzún, C., Huygens, D., y Boeckx, P. 2009. Temperate ecosystems of Chile: Characteristic biogeochemical cycles and disturbance regimes. In Ecological advances on Chilean temperate rainforests. Verhoest N, P Boeckx, C Oyarzún, R Godoy (eds.). Academia Press, Belgium, p. 31-40.

González-Polo, M., Fernández-Souto, A., y Austin, A.T. 2013. Coarse Woody Debris Stimulates Soil Enzymatic Activity and Litter Decomposition in an Old-Growth Temperate Forest of Patagonia, Argentina. Ecosystems, 16(6): 1025-1038.

Gough, C.M., Vogel, C.S., Kazanski, C., Nagel, L., Flower, C.E., y Curtis, P.S. 2007. Coarse woody debris and the carbon balance of a north temperate forest. Forest Ecology and Management, 244: 60-67.

Heinemann, K., y Kitzberger, T. 2006. Effects of position, understory vegetation and coarse woody debris on tree regeneration in two environmentally contrasting forests of north-western Patagonia: a manipulative approach. Journal of Biogeography, 33(8): 1357-1367.

Huygens D, P Boeckx, P Templer, L Paulino, O Van Cleemput, C Oyarzún, C Muller, R Godoy. 2008. Mechanisms for retention of bioavailable nitrogen in volcanic rainforest soil. Nature Geoscience, 1: 543-548.

Ibarra M, J Caldentey, A Promis. 2011. Descomposición de hojarasca en rodales de Nothofagus pumilio de la región de Magallanes. Bosque, 32(3): 227-233.

Jia-bing, W., De-xin, G., Shi-jie, H., Mi, Z., y Chang-jie, J. 2005. Ecological functions of coarse woody debris in forest ecosystem. Journal of Forestry Research, 16(3): 247-252. 
Lombardi, F., Cocozza, C., Lasserre, B., Tognetti, R., y Marchetti, M. 2011. Dendrochronological assessment of the time since death of dead wood in an old growth Magellan's beech forest, Navarino Island (Chile). Austral Ecology, 36(3): 329-340.

Marra, J.L., y Edmonds, R.L. 1994. Coarse woody debris and forest floor respiration in an old-growth coniferous forest on the Olympic Peninsula, Washington, USA. Canadian Journal of Forest Research, 24(9): 1811-1817. Oyarzún, C.E., Godoy, R., Schrijver, A., Staelens, J., y Lust, N. 2004. Water chemistry and nutrient budgets in an undisturbed evergreen rainforest of southern Chile. Biogeochemistry, 71: 107-123.

Schlegel, B.C., y Donoso, P.J. 2008. Effects of forest type and stand structure on coarse woody debris in Oldgrowth rainforests in the Valdivian Andes, south-central Chile. Forest Ecology and Management, 255: 19061914.

Spies, T.A., Franklin, J.F. y Thomas,T.B. 1988. Coarse woody debris in Douglas-fir forest of western Oregon and Washington. Ecology, 69: 16891702.

Veblen TT, AH Holz, J Paritsis, E Raffaele, T Kitzberger, M Blackhall. 2011. Adapting to global environmental change in Patagonia: What role for disturbance 38ecology?. Austral Ecology, 36:891-903.

Watkinson, S.C., Bebber, D., Darrah, P.R., Fricker, M.D., Tlalka, M., y Boddy, L. 2006. The role of wood decay fungi in the carbon and nitrogen dynamics of the forest floor. In Fungi in biogeochemical cycles. GM Gadd (Eds). Cambridge University Press. Cambridge, UK. pp. 151-181.

Yan, E., Wang, X., y Huang, J. 2006. Concept and Classification of Coarse Woody Debris in Forest Ecosystems. Frontiers of Biology in China, 1(1):76-84. 